\title{
Inconsistency of self-schematic content in moderately depressed college students
}

\author{
MICHAEL J. ROSS \\ Saint Louis University, St. Louis, Missouri \\ and \\ JOHN H. MUELLER \\ University of Missouri-Columbia, Columbia, Missouri
}

\begin{abstract}
Moderately depressed and nondepressed subjects rated positive- and negative-trait adjectives in terms of self-descriptiveness or other-descriptiveness across three presentations of the words. For both the self- and other-referenced tasks, moderately depressed subjects rated negative words more inconsistently across the three presentations than did nondepressed subjects. In addition, depressed subjects demonstrated enhanced recognition of words that produced inconsistent ratings, relative to nondepressed subjects.
\end{abstract}

Recent research on self-schematic processing has indicated that the consistency with which persons view themselves is influenced by their degree of depressive symptomatology. For example, MacDonald and Kuiper (1984) found that severe depressives consistently endorsed negative-trait adjectives as self-descriptive, whereas nondepressed subjects were consistent for positive selfdescriptors. Mild depressives, on the other hand, have been shown to be inconsistent when making judgments of self-descriptiveness (MacDonald, Kuiper, \& Olinger, 1985; Ross, 1989). Moreover, the mild depressives' inconsistency extended to both positive and negative aspects of self (Ross, 1989). However, there have been no investigations of the consistency of self-schema content in moderately depressed subjects. In this context, "moderate" refers to subjects who are more depressed than those labeled "mild," but are less depressed than those labeled "severe."

Developmental models of self-schematic content in depression (e.g., Davis, 1979; Kuiper, Derry, \& MacDonald, 1982) suggest that the moderately depressed person lacks the consolidated positive self-schema of the nondepressive, as well as the consolidated negative self-schema of the severe depressive. Consequently, moderate depressives, relative to nondepressed subjects, should demonstrate greater inconsistency in self-descriptiveness judgments. However, whether moderately depressed persons are inconsistent regarding the positive aspects of themselves, the negative aspects of themselves, or both, remains unknown.

Preparation of this article was supported by a Beaumont Faculty Development Grant from Saint Louis University to the first author. Portions of this article were presented earlier at a meeting of the Midwestern Psychological Association. Appreciation is extended to Paul J. Handal for his helpful comments. Requests for reprints should be sent to Michael J. Ross, Department of Psychology, Saint Louis University, 221 N. Grand Blvd., St. Louis, MO 63103.
Current theories of self-functioning assume that memory benefits that normally occur when the self-schema is activated during encoding will be diminished if the content is absent from the self-concept. Ross (1989) found a relationship between self-schematic inconsistency and cognitive processing in a mildly depressed sample. Specifically, mild depressives demonstrated processing deficits (i.e., reduced recall) for inconsistent self-schema content, although these findings were reliably associated only with negative traits. If the schematic content of moderate depressives is inconsistent, then it too should be associated with cognitive-processing deficits. However, whether content-specificity for inconsistent features exists in this population is unknown.

Finally, none of these studies included an investigation of consistency for nonself judgments. Cognitive models of depression (e.g., Beck, 1967) suggest that severe depressives view both themselves and their environment in a consistently negative fashion. Therefore, moderate depressives should demonstrate inconsistency for both self and others.

The present study was designed to address each of these limitations in the empirical literature on the consistency of self-schema content in depression. Specifically, moderately depressed and nondepressed subjects were compared in terms of the number of inconsistent self-descriptiveness and other-descriptiveness ratings across three presentations of positive- and negative-trait adjectives, and in terms of recognition for those words rated inconsistently.

\section{METHOD}


ogy course who had scored at the lower and upper extremes on the Dempsey D-30, compared to more than 800 of their classmates. The D-30 had been administered several weeks before the actual experiment.

Thirty-two subjects (19 males, 13 females) who scored high on the D-30 and who also subsequently scored above 13 on the BDI at the time of the actual experiment comprised the depressed group. The nondepressed group consisted of 40 subjects ( 21 males, 19 females) who scored low on the D-30 and who scored less than 5 on the BDI. Mean D-30 scores were 17.1 for the depressed group and 1.3 for the nondepressed group; mean BDI scores were 20.6 for the depressed group and 1.3 for the nondepressed group. The mean BDI scores for each group corresponded to Beck's (1967) recommended cutoff scores for classification as moderately depressed (i.e., 16-23) and nondepressed (i.e., 0-9).

\section{Materials}

The slides presented during the recognition-study phase consisted of the 64 trait adjectives previously used by Derry and Kuiper (1981). Normative ratings (see Kuiper et al., 1982, pp. 83-85) determined these words to be equivalent in terms of imagery value, word length, and word frequency, but differentiated in terms of affective valence, with half being depressive/negative and the other half being nondepressive/positive. The slides were divided into two sets of 32 each ( 16 positive and 16 negative adjectives in each set), and each set was used as the target and distractor set across subjects.

\section{Procedure}

During the study phase, half of the single-adjective slides in the rating set were presented once and the other half were presented three times. Thus, each subject saw one of the two 32-item sets consisting of 16 adjectives ( 8 positive, 8 negative) which were presented once, and 16 adjectives ( 8 positive, 8 negative) which were presented three times, such that each subject rated a total of 64 randomly ordered single-adjective slides. Each slide was presented at a 5-sec rate, and the subject rated the adjective on a $1-5$ scale $(1=$ low; $5=$ high $)$ according to their randomly assigned decision condition. Half the subjects in each group rated the words for self-descriptiveness; half the subjects rated the words in terms of descriptiveness of their best friend.

Following the study phase, the subjects were administered the Buss Self-Consciousness Scale (Fenigstein, Scheier, \& Buss, 1975), a 23-item questionnaire answered on a 5-point scale. This questionnaire was only used as a filler task to limit the effects of short-term memory on the subsequent recognition test and to minimize ceiling effects; the results were not scored.

The subjects were then presented with a previously unannounced test of incidental learning in which the 32 words from the study phase and the 32 words from the other study set that had not been presented previously were randomly mixed together and presented one at a time at a 5-sec rate. The subjects indicated on a separate answer sheet whether each single-adjective slide was old (i.e., was presented during the study phase) or new (i.e., was not presented during the study phase).

\section{RESULTS}

Analysis of decision inconsistency required omitting words that were presented only once during the study task. Each item presented three times was then classified in terms of the combined results of its decision-consistency and self-descriptiveness rating. This resulted in three consistency-descriptiveness item categories: consistentdescriptive (3-Yes), consistent-nondescriptive (3-No), and inconsistent (2-Yes, 1-No or 1-Yes,2 No). For purposes of analyses, items rated 1-2 were considered nondescriptive, and those rated 3-5 were considered descriptive.

\section{Endorsement Patterns}

To test the hypothesis of increased decision inconsistency of positive- and negative-trait adjectives as a func- tion of depression, a $2 \times 2 \times 2$ (depression group $\times$ task $x$ word content) analysis of variance (ANOVA) of only the inconsistent ratings was performed (i.e., omitting the 3-Yes and 3-No words). A significant main effect for word content $[F(1,68)=10.40, p<.002]$ was qualified by a significant depression $\times$ word content interaction $[F(1,68)=5.96, p<.02]$. These results indicate that depressed subjects generated a greater number of inconsistent ratings than did nondepressed subjects for negative words ( $M s=1.01$ vs. 0.51 , respectively), but the two groups did not differ in terms of inconsistent ratings of positive words ( $M s=0.52$ vs. 0.42 , respectively).

The data support the hypothesis that moderately depressed subjects would demonstrate increased inconsistency for both self and others. First, there was no statistically significant interaction between depression and task in terms of the number of inconsistent ratings $[F(1,68)=0.34, p<.56]$. In addition, planned comparisons on the means generated from the nonsignificant depression $\times$ task $\times$ word content interaction revealed that the depressives' enhanced inconsistency was associated with negative information and not with positive information related to both self $(M \mathrm{~s}=1.08 \mathrm{vs.} 0.76$, respectively) and others $(M s=0.93$ vs. 0.29 , respectively).

\section{Recognition}

Since the pooling procedures resulted in a different number of "yes" items for each of the within-subject factors, the recognition data were transformed to hit rates in order to correct for such variation. The signal-detection measure, $d^{\prime}$, was then computed for analysis, since this metric takes into account covariation in hit rate and falsealarm rate.

A $2 \times 2 \times 2$ (depression group $\times$ task $\times$ word content) ANOVA for inconsistent ratings was performed to test the hypothesis of reduced strength of processing (lower recognition) as a function of depression and inconsistency. Although there was a significant main effect for the depression group $[F(1,40)=4.78, p<.03]$, the results were in the opposite direction from the hypothesis; that is, depressed subjects demonstrated enhanced recognition of inconsistent ratings relative to nondepressed subjects ( $M$ s $=3.75$ vs. 3.41 , respectively).

\section{DISCUSSION}

Compared to normal subjects, moderate depressives showed greater inconsistency in rating negative traits, but not in rating positive traits, for descriptiveness of self and a familiar other. Moderate depressives also demonstrated enhanced recognition of words rated inconsistently, compared to nondepressives.

These results extend previous findings (e.g., MacDonald et al., 1985; Ross, 1989) of inconsistency in self-schematic content within subclinical depressed populations. Whereas previous research with a mildly depressed sample has indicated inconsistency for both the positive and the negative aspects of self (Ross, 1989), the present sample of moderately depressed persons limited their inconsistency to negative referents. Taken together, these studies suggest that increased severity of subclinical depression is associated with greater consistency (but lower numbers) of positive features of the self-concept, whereas negative self-descriptors remain inconsistent and uncertain. In addition, the fact that moderate 
depressives demonstrated inconsistency for negative traits associated both with themselves and with their best friends provides support for Beck's (1967) notion of a cognitive triad in depressive symptomatology (i.e., negative evaluations of self, environment, and the future).

The fact that moderately depressed subjects demonstrated enhanced recognition for inconsistent schematic information was contrary to our prediction. Previous findings by Ross (1989) with a subclinically, but less severely, depressed sample indicated that inconsistent self-schematic content was associated with less efficient and less powerful cognitive processing. For the moderate depressive, however, it appears that inconsistent schematic content is associated with enhanced cognitive processing. It may be that the negative information, although still not fully incorporated into the self-image, becomes more salient as depression deepens. However, this conclusion must be tempered by differences between the two studies. Aside from differences in the severity of the depressed samples between the two studies (i.e., mild vs. moderate), Ross's study used recall as the dependent measure, whereas the present study used recognition performance. It is suggested that further research on inconsistency in schematic processing with depressed populations use similar dependent measures and compare groups representing varying levels of depression severity directly in the same study with the same procedures.

\section{REFERENCES}

Beck, A. T. (1967). Depression: Clinical, experimental, and theoretical aspects. New York: Harper \& Row.
BECK, A. T. (1978). Depression inventory. Philadelphia: Center for Cognitive Therapy.

Davis, H. (1979). Self-reference and the encoding of personal information in depression. Cognitive Therapy \& Research, 3, 97-110.

DEMPSEY, P. A. (1964). A unidimensional depression scale for the MMPI. Journal of Consulting Psychology, 28, 364-370.

DERRY, P. A., \& KUIPER, N. A. (1981). Schematic processing and selfreference in depression. Journal of Abnormal Psychology, 90, 286-297.

Fenigstein, A., Scheier, M. F., \& Buss, A. H. (1975). Public and private self-consciousness: Assessment and theory. Journal of Consulting \& Clinical Psychology, 43, 522-527.

Kuiper, N. A., Derry, P. .A., \& MacDonald, M. R. (1982). Selfreference and person perception in depression. In G. Weary \& H. Mirels (Eds.), Integrations of clinical and social psychology (pp. 79-103). New York: Oxford University Press.

MacDonald, M. R., \& Kuiper, N. A. (1984). Self-schema decision consistency in clinical depressives. Journal of Social \& Clinical Psychology, 2, 264-272.

MacDonald, M. R., Kuiper, N. A., \& Olinger, L. J. (1985). Vulnerability to depression, mild depression, and degree of schema consolidation. Motivation \& Emotion, 9, 369-379.

Ross, M. J. (1989). Self-schematic instability in mild depression. Journal of Social Behavior \& Personality, 4, 119-126.

(Manuscript received February 27, 1989.) 